\title{
Molecular Modeling Studies of Substituted 2,4,5-Trisubstituted Triazolinones Aryl and Nonaryl Derivatives as Angiotensin II AT Receptor Antagonists
}

\author{
Mukesh C. Sharma, ${ }^{1}$ D. V. Kohli, ${ }^{1}$ and Smita Sharma ${ }^{2}$ \\ ${ }^{1}$ Drug Research Laboratory, Department of Pharmaceutical Sciences, Dr. H. S. Gour University, Sagar 470 003, India \\ ${ }^{2}$ Department of Chemistry, Chaudher Dilip Singh Kanya Mahavidyalaya, Bhind 477001, India \\ Correspondence should be addressed to Mukesh C. Sharma; mukesh2206@rediffmail.com
}

Received 26 June 2012; Revised 21 October 2012; Accepted 21 October 2012

Academic Editor: Tomokazu Yoshimura

Copyright ( 2013 Mukesh C. Sharma et al. This is an open access article distributed under the Creative Commons Attribution License, which permits unrestricted use, distribution, and reproduction in any medium, provided the original work is properly cited.

\begin{abstract}
The development of new therapies to treat hypertension and cardiovascular diseases. A series of 2,4,5-trisubstituted triazolinones aryl and nonaryl derivatives were subjected to Group-based QSAR, $k$-nearest neighbour molecular field analysis, and pharmacophore mapping. Multiple linear regression (MLR) methodology coupled with feature selection method namely simulated annealing, was applied to derive Group based QSAR models which were further validated for statistical significance and predictive ability by internal and external validation. The best physicochemical descriptors, namely, R1chiV1, R2T_N_O_3, R2chlorines count, R2T_C_N_4, and R2SssNHE index, contribute significantly to the biological activity. The statistically significant best Groupbased QSAR model has $r^{2}=0.8357$ and $q^{2}=0.7266$ with pred_ $r^{2}=0.8138$. The 3D-QSAR studies were performed using the simulated annealing selection $k$-nearest neighbor molecular field analysis approach; a leave-one-out cross-validated correlation coefficient $q^{2}=0.7461$ and predicate activity pred_r $r^{2}=0.7790$ were obtained. Contour maps using this approach showed that steric, electrostatic, and hydrophobic effects dominantly determine binding affinities. Pharmacophore hypotheses were generated by the mol sign module and found to contain common features like hydrogen bond donor acceptor, donor, positive, negative ionizable, and hydrophobic features. This model can be used for preliminary screening of large number of substituted $3 \mathrm{H}-1,-2$,4 triazolinone aryl and nonaryl derivatives. The information rendered by 3D-QSAR models may lead to a better understanding of structural requirements of triazolinone aryl and nonaryl derivatives and also aid in designing novel potent antihypertensive molecules.
\end{abstract}

\section{Introduction}

The renin-angiotensin system (RAS) is considered to be the major regulator of blood pressure, electrolyte balance and renal, neuronal as well as endocrine functions related to cardiovascular control $[1,2]$. Activation of the renin-angiotensin cascade begins with renin secretion from the juxtaglomerular apparatus of the kidney and culminates in the formation of the octapeptide angiotensin II receptor which then interacts with specific receptors present in different tissues [3, 4]. The RAS is the key factor in most cases essential hypertension, as indicated by successes in treatment of hypertensive patients with various angiotensin I converting enzyme (ACE) inhibitors and receptor blockers. Renin was a central subject of intense investigation because of its role in blood pressure regulation before the discovery of angiotensinogen [1]. Two distinct subtypes of Ang II receptors, $\mathrm{AT}_{1}$ and $\mathrm{AT}_{2}$, have been identified by using non-peptidic, subtypeselective antagonists (losartan for $\mathrm{AT}_{1}$ and PD123319 for $\mathrm{AT}_{2}$, resp.). Although both receptor types belong to the G-protein-coupled receptor superfamily which has seven transmembrane spanning domains, the properties of the two Ang II receptors have been shown to be different $[5,6]$. The $\mathrm{AT}_{1}$ receptors undergo rapid internalization and desensitization upon agonist stimulation, whereas $\mathrm{AT}_{2}$ receptors do not internalize and desensitize [7]. A major function of the RAS is the regulation of blood pressure, and this effect has been of great interest to the pharmaceutical industry for several 
decades in attempts to produce antihypertensive drugs. The successful approaches have been to inhibit the hypertensive actions of angiotensin II by either inhibit the formation of angiotensin II using ACE inhibitors or to block it's binding to the $\mathrm{AT}_{1}$ receptor using antagonists [8]. The $\mathrm{AT}_{1}$ receptor mediates virtually all well-known physiological actions of Ang II, such as vasoconstriction, aldosterone release, water and salt retention, sympathetic transmission, cell growth, and proliferation [9]. $\mathrm{AT}_{1}$ receptors are primarily found in the brain, adrenal gland, vasculature, heart, and kidney [10]. The pioneering efforts of the DuPont Group have generated a promising first nonpeptide $\mathrm{AT}_{1}$ antagonist losartan, which represent the prototype of the sartans. All the angiotensin II receptor blockers bind to the $\mathrm{AT}_{1}$ receptors and share common some salient structural features. These sartans are designed to mimic the C-terminal part of angiotensin II receptor [11]. Quantitative structure-activity relationship (QSAR) approach is certainly useful for designing newer drugs when the target is not known or if there are multiple targets. Since physicochemical data are not always available to develop the predictive models; the only alternative is to utilise the theoretical molecular descriptors for such modelling purposes that can be derived solely from the chemical structures of compounds. The QSAR approach helps to correlate the specific biological activities or physical properties of a series of compounds with the measured or computed molecular properties of the compounds, in terms of descriptors [12, 13]. QSAR methodologies save resources and expedite the process of the development of new molecules and drugs. QSAR methods have been applied widely for drug discovery, lead optimization, risk assessment, toxicity prediction, and regulatory decisions. Hence, in continuation to our efforts $[11,14-50]$ in developing QSAR studies for angiotensin II receptor antagonists. In this investigation, multiple linear regression (MLR) methodology, coupled with feature selection simulated annealing, was applied to derive QSAR models which were further validated for statistical significance and predictive ability by internal and external validation. The generated models provide insight into the influence of various interactive fields on the activity and, thus, can help in designing and forecasting the activity of novel antihypertensive molecules.

\section{Materials and Methods}

2.1. Data Set for Analysis. In the present study, a series of $3 \mathrm{H}-1,-2,-4$ triazolinone aryl and nonaryl substituents (Table 1) having inhibitory potency towards angiotensin II $\mathrm{AT}_{1}$ receptor antagonists was used as the model data set [51]. The biological activity values $\left[\mathrm{IC}_{50}\right]$ reported in the literature were converted to their molar units and then converted to negative logarithmic scale $\left(\mathrm{pIC}_{50}\right)$ and subsequently used as the dependent variable for the QSAR analysis. Table 1 shows the structure of 55 such compounds along with their biological activity values. The strategy for the selection of compounds to be included in the test set was a random selection of compounds that exhibited a varied range of inhibitory activities and structural diversities.
2.2. Molecular Structure Generation. The molecular structure of all the 55 molecules was sketched using VLife MDS 3.5 software [52] in the $2 \mathrm{D}$ builder module and then the structures were converted to 3D space for further analysis. All the compounds were batch optimized for the minimization of energies and geometry optimization using Merck molecular force field (MMFF) followed by considering distancedependent dielectric constant of 1.0, convergence criterion or root-mean-square (RMS) gradient at $0.01 \mathrm{kcal} / \mathrm{mol} \AA$, and the iteration limit to 10,000 [53].

2.3. Selection of Training and Test Set. The dataset of 55 molecules was divided into training and test set by sphere exclusion (SE) method with $\mathrm{pIC}_{50}$ activity field as dependent variable and various $3 \mathrm{D}$ descriptors calculated for the compounds as independent variables. Selection of molecules in the training set and test is a key and important feature of any QSAR model. Therefore, care was taken in such a way that biological activities of all compounds in test set lie within the maximum and minimum value range of biological activities of training set of compounds. The sphere exclusion method [54, 55] was adopted for division of training and test data set comprising of 40 and 15 molecules, respectively, with a dissimilarity value of 16.8 . Sphere exclusion method was adopted for division of training and test set. This is a rational selection method which takes into consideration both biological and chemical space for division of dataset. Dissimilarity value provides handle to vary train/test set size. It needs to be adjusted by trial and error until a desired division of train and test set is achieved. As a rule, increase in dissimilarity value will lead to increase in number of molecules in the test set.

2.4. Group-Based QSAR. Group-based QSAR allows establishing a correlation of chemical group/fragment variation at different molecular sites of interest with the biological activity. The proposed Group-basedQSAR methodology allows ease of interpretation unlike any conventional QSAR method which could only suggest important descriptors but does not reflect the site where it has to be optimized for design of new molecules [56, 57]. Fragmentation is done by applying specific chemical rules for breaking the molecules along specific bonds and/or bonds on ring fusion and/or any pharmacophoric feature such as hydrogen bond acceptor, hydrogen bond donor, hydrophobic group, charged group, and so forth.

Group-based descriptors were calculated for various groups present at different substitution sites of the molecules (i.e., Fragments R1, R2, and R3). The removal of the invariable group descriptors resulted in a total of 480 group descriptors which can be used further. Since the same descriptors are calculated for various groups at different sites, the following nomenclature is used for naming a descriptor at a particular position, for example R1_smr represents the molar refractivity of the group present at substitution site R1. Established 2D descriptors like chi indices, valencebased chi indices, electrotopological indices, Baumann alignment independent topological descriptors, rotatable bonds, 
TABLE 1: The structures of triazolinone aryl and nonaryl derivatives with their activities.

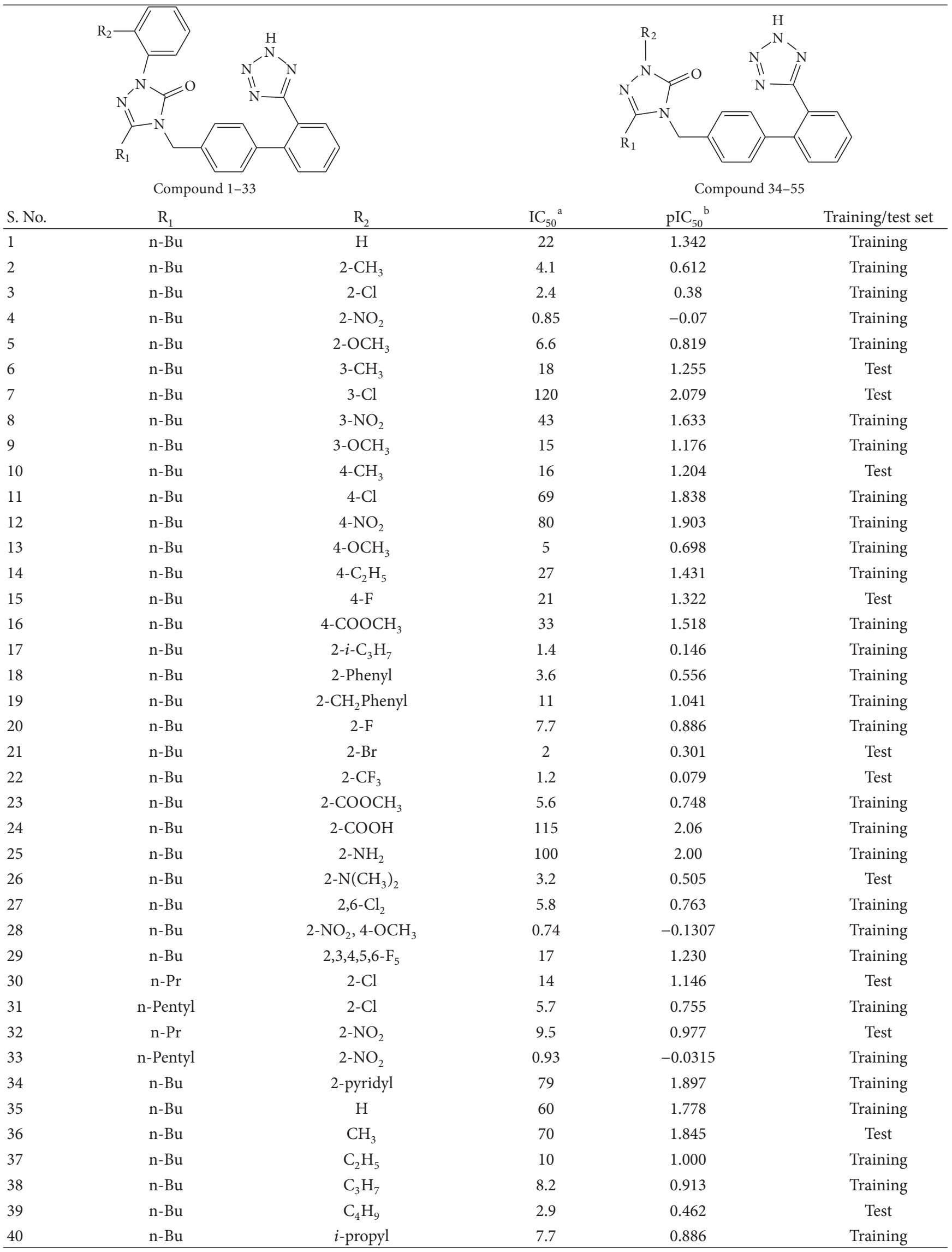


TABLe 1: Continued.

\begin{tabular}{|c|c|c|c|c|c|}
\hline \multirow[b]{2}{*}{ S. No. } & $\mathrm{R}_{2} \longrightarrow$ & & \multirow[b]{2}{*}{$\mathrm{IC}_{50}{ }^{\mathrm{a}}$} & & \\
\hline & $\begin{array}{l}\text { Compound 1-33 } \\
\mathrm{R}_{1} \\
\end{array}$ & & & $\mathrm{pIC}_{50}{ }^{\mathrm{b}}$ & Training/test set \\
\hline 41 & $\mathrm{n}-\mathrm{Bu}$ & $i$-butyl & 3.2 & 0.505 & Test \\
\hline 42 & $\mathrm{n}-\mathrm{Bu}$ & $s$-butyl & 1.8 & 0.255 & Training \\
\hline 43 & $\mathrm{n}-\mathrm{Bu}$ & $\mathrm{CH}_{2} \mathrm{COOCH}_{3}$ & 17 & 1.23 & Training \\
\hline 44 & $\mathrm{n}-\mathrm{Bu}$ & Benzyl & 4.6 & 0.662 & Test \\
\hline 45 & $\mathrm{n}-\mathrm{Bu}$ & $\mathrm{CH}_{2}$ (c-Hexane) & 2.9 & 0.462 & Training \\
\hline 46 & $\mathrm{n}-\mathrm{Bu}$ & $\left(2-\mathrm{CH}_{3}\right)$ benzyl & 11 & 1.401 & Test \\
\hline 47 & $\mathrm{n}-\mathrm{Bu}$ & $\left(3-\mathrm{CH}_{3}\right)$ benzyl & 12 & 1.079 & Training \\
\hline 48 & $\mathrm{n}-\mathrm{Bu}$ & $\left(\alpha-\mathrm{CH}_{3}\right)$ benzyl & 4.9 & 0.69 & Training \\
\hline 49 & $\mathrm{n}-\mathrm{Bu}$ & $\left(2-\mathrm{COOCH}_{3}\right)$ benzyl & 11 & 1.041 & Test \\
\hline 50 & $\mathrm{n}-\mathrm{Bu}$ & $\left(3-\mathrm{COOCH}_{3}\right)$ benzyl & 110 & 2.041 & Training \\
\hline 51 & $\mathrm{n}-\mathrm{Bu}$ & $\left(4-\mathrm{COOCH}_{3}\right)$ benzyl & 23 & 1.361 & Training \\
\hline 52 & $\mathrm{n}-\mathrm{Bu}$ & $\left(\alpha-\mathrm{COOCH}_{3}\right)$ benzyl & 12 & 1.079 & Training \\
\hline 53 & $\mathrm{n}-\mathrm{Bu}$ & (2-COOH) benzyl & 490 & 2.690 & Training \\
\hline 54 & $\mathrm{n}-\mathrm{Bu}$ & $(\alpha-\mathrm{COOH})$ benzyl & 68 & 1.832 & Training \\
\hline 55 & $\mathrm{n}-\mathrm{Bu}$ & $\mathrm{CH}_{2} \mathrm{C}_{6} \mathrm{~F}_{6}$ & 6 & 1.505 & Training \\
\hline
\end{tabular}

${ }^{\mathrm{a}} \mathrm{IC}_{50}$ or inhibition of specific binding of [ ${ }^{125} \mathrm{I}$-rabbit aorta Ang II AT $\mathrm{A}_{1}$ receptor.

${ }^{\mathrm{b}}-\log \mathrm{IC}_{50}$ to generate equation.

and/or other 3D alignment independent descriptors like dipole moment, Polar Surface Area (PSA), and so forth for various fragments present in each molecule in the series. In this study, a simulated annealing coupled with multiple linear regression SA-MLR resulted in a significant G-QSAR model with descriptors.

\subsection{D QSAR Studies Using $k$-Nearest Neighbour}

2.5.1. Alignment of Molecules. The molecular alignment utility can be used to study the shape variation with respect to the base structure selected for alignment. This is an attempt to identify the best overlapping between the structures of molecules. The most critical input for the 3D-QSAR modeling is the alignment of the molecules. Energy minimized and geometry-optimized structures of molecules were aligned by the template-based method [58], where a template structure is defined and used as a basis for alignment of a set of molecules, and a reference molecule is chosen on which the other molecules of the data set get aligned considering the chosen template. The template structure, that is, triazolinone ring, was used for the alignment by considering the common elements of the series as shown in Figure 1(a). The reference molecule is chosen in such a way that it is the most active among the series of molecules considered. The compound
28 possessed very high angiotensin $\mathrm{II}_{\mathrm{AT}_{1}}$ receptor activity which made it a valid lead molecule and, therefore, was chosen as a reference molecule. After optimising, the template structure and the reference molecule were used to superimpose all molecules from the series using the template alignment method in VLife MDS 3.5 software [52] to obtain optimal alignment between the molecular structures necessary for ligand-receptor interactions. The superimposition of all molecules based on minimising RMS deviation is shown in Figure 1(b).

Molecular fields are the steric, electrostatic, and hydrophobic fields interaction energies which are used to formulate a relationship between steric and electrostatic properties together with the biological activities of compounds. Each conformation is taken in turn and the molecular fields around it are calculated. This is done by generating 3D rectangular grids around the molecule and calculating the interaction energy between the molecule and probe group placed at each grid point. The major steps of molecular field analysis are (1) generating conformers and energy minimization; (2) matching atoms with template structure and aligning molecules against reference molecule using default option; (3) setting MFA preferences (rectangular grid with $2 \AA$ step size, charges by MMFF, $\mathrm{CH}_{3}$ as probe); (4) creating the field [59]. 


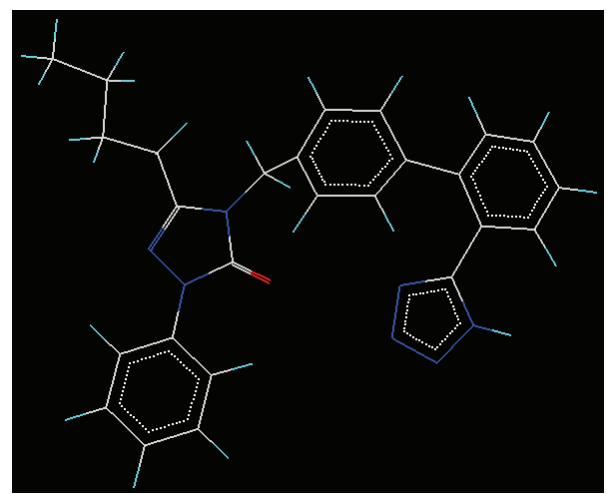

(a)

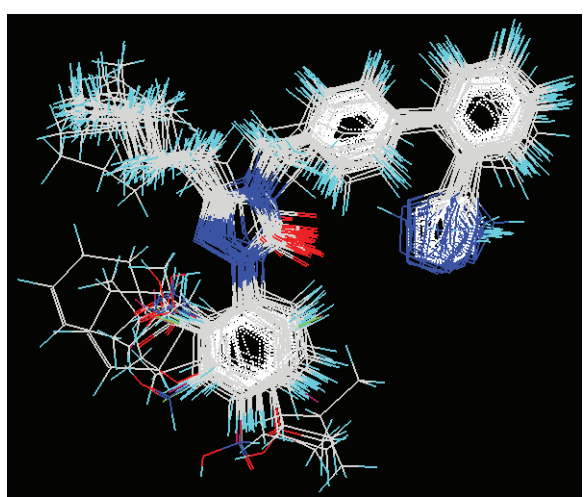

(b)

FIgURE 1: (a) Template for the superposition of the rest of the molecules. (b) Stereoview of all the aligned molecules.

2.5.2. Calculation of $3 D$ Descriptors. To derive the $k$-nearest neighbour-MFA descriptor fields, a 3D cubic lattice with grid spacing of $2 \AA$ in $x, y$, and $z$ dimensions was created to encompass the aligned molecules. Using Tripos force field [60] and Gasteiger and Marsili charge type [61], electrostatic, steric and hydrophobic field descriptors were calculated. The dielectric constant was set to 1.0 considering the distance dependent dielectric function probe setting was carbon atom with charge 1.0. This resulted in calculation of 3600 field descriptors (1,200 for each electrostatic, steric and hydrophobic) for all the compounds in separate columns. They represent area in space where steric, electrostatic, and hydrophobic field interactions are responsible for the observed variation of the biological activity.

\subsection{3. $k$-Nearest Neighbour with Simulated Annealing Method.} 3DQSAR studies were carried out by $k$-nearest neighbour method using simulated annealing method. The $k$-nearest neighbour methodology relies on a simple distance learning approach whereby an unknown member is classified according to the majority of its $k$-nearest neighbours in the training set. The standard kNN method is implemented simply as follows:

(1) calculate the distances between an unknown object $(u)$ and all the objects in the training set;

(2) select $k$ objects from the training set most similar to object $u$, according to the calculated distances; and

(3) classify object $u$ with the group to which the majority of the $k$ objects belongs $[62,63]$. An optimal $k$ value is selected by optimization through the classification of a test set of samples or by leave-one-out crossvalidation.

The variables and optimal $k$ values were chosen using stepwise variable selection method. This method employs a stepwise variable selection procedure combined with $\mathrm{kNN}$ to optimize

(i) the number of nearest neighbors $(k)$ and

(ii) the selection of variables from the original pool.
The step-by-step search procedure begins by developing a trial model with a single independent variable and adds independent variables, one step at a time, examining the fit of the model at each step (using weighted kNN cross-validation procedure). The method continues until there are no more significant variables remaining outside the model. Once the training and test sets were generating, $\mathrm{kNN}$ methodology was applied to the descriptors generated over the grid. The steric, electrostatic, and hydrophobic energies were computed at the lattice points of the grid using a methyl probe of charge +1 . These interaction energy values were considered for relationship generation and utilized as descriptors to decide the nearness between molecules.

Simulated annealing [64] is the simulation of a physical process, "annealing," which involves heating the system to a high temperature and then gradually cooling it down to a preset temperature (e.g., room temperature). During this process, the system samples possible configurations distributed according to the Boltzmann distribution so that at equilibrium, low energy states are the most populated [65]. The Simulated annealing method employs the $k$-nearest neighbour classification principle combined with the simulated annealing variable selection procedure. The simulated annealing $k$-nearest neighbour-MFA can be summarized as follows.

(i) Generate a trial solution to the underlying optimization problem; that is, a $k$-nearest neighbourMFA model is built based on a random selection of descriptors.

(ii) Calculate the value of the fitness function, which characterizes the quality of the trial solution to the underlying problem, that is, the $q^{2}$ value for a $k$ nearest neighbour-MFA model.

(iii) Perturb the trial solution to obtain a new solution, that is, change a fraction of the current trial solution descriptors to other randomly selected descriptors and build a new $k$-nearest neighbour-MFA model for the new trial solution.

(iv) Calculate the value of the fitness function $\left(q^{2}\right.$ new) for the new trial solution. 
(v) Apply the optimization criteria: if $q^{2}$ curr $\leq q^{2}$ new the new solution is accepted and used to replace the current trial solution; if $q^{2}$ curr $>q^{2}$ new, the new solution is accepted only if the Metropolis criterion is satisfied; that is,

$$
\text { rnd }<e^{-\left(q^{2} \text { curr }-q^{2} \text { new }\right) / T},
$$

where rnd is a random number uniformly distributed between 0 and 1 and $T$ is a parameter analogous to the temperature in the Boltzmann distribution.

(vi) Steps 3-5 are repeated until the termination condition is satisfied. The temperature-lowering scheme and the termination condition used in this work have been adapted $[66,67]$ Thus, when a new solution is accepted or when a preset number of successive steps of generating trial solutions (20 steps) do not lead to a better result, the temperature is lowered by $10 \%$ (the default initial temperature is $1000 \mathrm{~K}$ ). The calculations are terminated, when either the current temperature of simulations reaches $10^{-6} \mathrm{~K}$ or the ratio between the current temperature and the temperature corresponding to the best solution found equal $10^{-6}$.

The Group based QSAR and 3D k-nearest neighbour model was generated by multiple linear regressions. It relates the dependent variable $Y$ (biological activity) to a number of independent variables $X$ (molecular descriptor) by using linear equations. This method of regression estimates the values of the regression coefficients by applying least square curve fitting method. MLR is based on least squares: the model is fit such that sum-of-squares of differences of observed and a predicted value is minimized. Multiple regressions are the standard method for multivariate data analysis. It is also called as ordinary least squares regression (OLS). This method of regression estimates the values of the regression coefficients by applying least squares curve fitting method. For getting reliable results, dataset having typically five times as many data points (molecules) as independent variables (descriptors) is required [68]. The regression equation takes the following form:

$$
Y=b 1 * x 1+b 2 * x 2+b 3 * x 3+c,
$$

where $Y$ is the dependent variable, the " $b$ "s are regression coefficients for corresponding " $x$ "s (independent variable), and " $c$ " is a regression constant or intercept.

2.5.4. Cross-Validation. Internal validation was carried out using leave-one-out $\left(q^{2}, \mathrm{LOO}\right)$ method [69]. To calculate $q^{2}$, each molecule in the training set was sequentially removed, the model refit using same descriptors, and the biological activity of the removed molecule predicted using the refit model. The $q^{2}$ was calculated using

$$
q^{2}=1-\frac{\sum\left(y_{i}-\widehat{y}_{i}\right)^{2}}{\sum\left(y_{i}-y_{\text {mean }}\right)^{2}},
$$

where $y_{i}$ and $\widehat{y}_{i}$ are the actual and predicted activity of the $i$ th molecule in the training set, respectively, and $y_{\text {mean }}$ is the average activity of all molecules in the training set. For external validation, activity of each molecule in the test set was predicted using the model generated from the training set. The pred_r $r^{2}$ value is calculated as follows:

$$
\text { pred_r } r^{2}=1-\frac{\sum\left(y_{i}-\widehat{y}_{i}\right)^{2}}{\sum\left(y_{i}-y_{\text {mean }}\right)^{2}},
$$

where $y_{i}$ and $\widehat{y}_{i}$ are the actual and predicted activity of the $i$ th molecule in the test set, respectively, and $y_{\text {mean }}$ is the average activity of all molecules in the training set. Both summations are over all molecules in the test set. Thus the pred_ $r^{2}$ value is indicative of the predictive power of the current model based on the external test set. The robustness of the models for training sets was examined by comparing these models to those derived for random data sets.

2.5.5. Evaluation of Models. The developed QSAR models are evaluated using the following statistical measures: $n$, the number of compounds in regression; $k$, number of variables; DF, degree of freedom; $r^{2}$, the squared correlation coefficient, $F$-test (Fischer's value) for statistical significance; $q^{2}$, crossvalidated correlation coefficient; pred_r $r^{2}, r^{2}$ for external test set; value in the randomization test. The regression coefficient $r^{2}$ is a relative measure of fit by the regression equation. For predicting the activity of a molecule, regression methods use the following equation:

$$
\text { Activity }=C_{O}+C_{1} D_{1}+C_{2} D_{2}+\cdots+C_{N} D_{N}
$$

where $C_{i}$ s are coefficients and $D_{i} s$ are descriptors. In the case of the kNN-MFA method, the activity of a molecule is predicted using

$$
\text { Activity }=C_{1} A_{1}+C_{2} A_{2}+\cdots+C_{k} A_{k},
$$

where $C_{i}$ s are weights and $A_{i} s$ are activities of the $k$-nearest neighbours in the training set. The nearest neighbours of any molecule are obtained from calculating the distance between the descriptors selected from various variable selection methods [68]. Thus, $k$-nearest neighbour MFA prediction uses an interpolative method, and hence predicted activities of new designed molecules will be within the range of activities of molecules in training set. Since the $k$-nearest neighbour method is based on distances of descriptors, their interpretation is quite difficult compared to the regression models.

2.6. Pharmacophore Mapping Studies. A pharmacophore model is a set of three-dimensional features that are necessary for bioactive ligands. Thus, it makes sense to align molecules based on features that are responsible for bioactivity. This study was performed using the software package VLife MDS $3.5 \mathrm{~mol}$ sign module is used for the identification, generation and analysis of pharmacophore by aligning small organic molecules based on their 3D pharmacophore features. All 55 aligned molecules were taken for pharmacophore development. Select the most active molecule to set it as reference. 
TABLE 2: Unicolumn statistics of training and test set for QSAR study.

\begin{tabular}{lccccc}
\hline Set & Average & Max & Min & Std. Dev & 0.6398 \\
Training & 4.7921 & 6.8403 & 5.2847 & 0.6382 & 131.547 \\
Test & 4.2451 & 6.1024 & 5.1074 & 68.4793 \\
\hline
\end{tabular}

The reference molecule is the molecule on which the other molecules of the align dataset get aligned. For five point pharmacophore identification, tolerance limit is set up to $30 \AA$ and max distance allowed between two features, set the value to $5 \AA[68]$.

\section{Results and Discussion}

Group based-QSAR equations were selected by optimizing the statistical results generated along with variation of the descriptors in these models. A UniColumn statistics for training set and test set were generated to check correctness of selection criteria for trainings and test set molecules (Table 2). The maximum and minimum value in training and set were compared in a way that

(1) the maximum value of $\mathrm{pIC}_{50}$ of test set should be less than or equal to maximum value of $\mathrm{pIC}_{50}$ of training set,

(2) the minimum value of $\mathrm{pIC}_{50}$ of test set should be higher than or equal to minimum value of $\mathrm{pIC}_{50}$ of training set.

This observation showed that test set was interpolative and derived within the minimum-maximum range of training set. The mean and standard deviation of $\mathrm{pIC}_{50}$ values of sets of training and test provide insights to relative difference of mean and point density distribution of two sets. The mean of the test sets were higher than the train sets which indicates the presence of relatively more active molecules as compared to the inactive ones. To ensure a fair comparison, the same training and test sets were used for each model's development. Group based-QSAR models involving fragment descriptors and their interactions (cross terms) were developed. Some statistically significant Group based-QSAR models are chosen for discussion.

$$
\mathrm{pIC}_{50}=-0.3334( \pm 0.0345) \mathrm{R} 1 \mathrm{chiV} 1+0.4167( \pm 0.0698)
$$
R2T_N_O_3 $+0.8631( \pm 0.1763)$ R2 Chlorines Count $+0.4572( \pm 0.0123)$ R2 T_C_N_4 + $0.7922( \pm 0.1842)$ R2SssNHE-index.

$$
N_{\text {traning }}=40, N_{\text {test }}=15, \mathrm{DF}=38, r^{2}=0.8357, q^{2}=
$$

$0.7266, F$ test $=39.631, q^{2} \_$se $=0.4863$, pred_ $r^{2}=0.8138$, pred_ $r^{2}$ se $=0.3047$.

The statistically best significant model 1 shows good squared correlation coefficient $\left(r^{2}\right)$ of 0.8357 explains $83.57 \%$ variance in biological activity. This model also indicates statistical significance $>99.9 \%$ with $F$ values $F=39.631$. Cross-validated squared correlation coefficient of this model was 0.7266 , which shows the good internal prediction power of this model. The contributing descriptor in part- $R_{1}$ is molecular connectivity indices descriptors such as R1chiV1
( 34.93\%) which measures the valence molecular connectivity index of 1st order. The negative coefficient of the molecular connectivity index descriptors (ChiV1) in the models suggest that the increase in branching in the molecule and the presence of heteroatoms increases the activity. The most contributing descriptor in part- $\mathrm{R}_{2}$ is $\mathrm{R}^{2}$ Chlorines Count ( $13.77 \%)$, suggesting that the presence of substituent with chlorine on the $\mathrm{R}^{2}$ site ring position will lead to an increase in activity. The other contributing descriptor in part- $R_{2}$ is R2T_N_O_3, and R2SssNHE-index is directly contributing to the activity, suggesting that increase in these descriptors of fragment R2 may lead to an increase in the antihypertensive activity. This descriptor means the count of pair of any nitrogen atom and any oxygen atom separated by three bond distance, for example, $\mathrm{N}-\mathrm{C}-\mathrm{C}-\mathrm{C}-\mathrm{O}$. As a positive contributing descriptor ( 12\%), T_N_O_3 indicates the presence of triazolinone ring at the $\mathrm{R} 2$ position of the scaffold would lead to a positive effect on the antihypertensive activity. The next positive contributing ( $9.21 \%)$ descriptor T_2_N_4 is the number of atoms separated from the nitrogen atom by four bonds, indicating that the presence of substituents with nitrogen atoms (e.g., $\left.-\mathrm{NO}_{2},-\mathrm{N}\left(\mathrm{CH}_{3}\right)_{2}\right)$, at the ring in the $\mathrm{R}^{2}$ position will lead to a positive effect on the activity [11, 70]. The other descriptor contributing is R2SssNHE-index which shows the importance of suggesting that the increase in electronegative atom environment adjacent to indicated $-\mathrm{NH}$ atom (i.e., SssN type) would result $(\sim 10 \%)$ in increase in the activity at $R^{2}$ position. The activity Contribution chart for GQSAR model is shown in Figure 2(a) and plots of observed versus predicted values of $\mathrm{pIC}_{50}$ are shown in Figure 2(b). The predicted (LOO) activities of the compounds by the above models is shown in Table 3.

$\mathrm{pIC}_{50}=+0.6689( \pm 0.0117)$ R2 polar surface area excluding P \& S + $0.4681( \pm 0.0356) \mathrm{R} 1 \mathrm{H}$-Count $+0.2625( \pm 0.0011)$ R2T_2_N_4 -0.3689 $( \pm 0.1770)$ R2S $\log \mathrm{P}+0.0317$.

$$
N_{\text {traning }}=40, N_{\text {test }}=15, \mathrm{DF}=35, r^{2}=0.8286, q^{2}=
$$
$0.7618, F$ test $=35.3584, r^{2}$ se $=0.1236, q^{2}$ se $=0.1733$, pred_ $r^{2}=0.7905$, pred_r $r^{2} \mathrm{se}=0.3268, Z$ SScore $\mathrm{Q}^{2}=2.85744$, Best Rand $\mathrm{Q}^{2}=1.78893$.

Model 2 generated using the SA-MLR method with 0.7884 as the coefficient of determination $\left(r^{2}\right)$ was considered model using the same molecules in the test and training sets as in G-QSAR. The statistical models for angiotensin $\mathrm{AT}_{1}$ receptor activity with internal predictive power $\left(q^{2}=\right.$ 0.7618 ) of $76 \%$ and a predictivity for the external test set (pred_ $r^{2}=0.7905$ ) of about $79 \%$. The contributing descriptor in part- $\mathrm{R}_{1}$ is $\mathrm{H}$-Count is a type of element count descriptor showing the number of hydrogen atoms in a compound, suggesting double, triple, or aromatic substituent over alkyl substituent of fragment R1 may lead to an increase in 
TABLE 3: Comparative observed and predicted activities (LOO) of triazolinone aryl and nonaryl by best 3D-QSAR models.

\begin{tabular}{|c|c|c|c|c|c|c|c|c|c|}
\hline \multirow{2}{*}{ Com } & \multirow{2}{*}{$\mathrm{pIC}_{50}$} & \multicolumn{2}{|c|}{ G-QSAR model 1} & \multicolumn{2}{|c|}{ G-QSAR model 2} & \multicolumn{2}{|c|}{ G-QSAR model 3} & \multicolumn{2}{|c|}{ SA-kNN model 4} \\
\hline & & Pred. & Res. & Pred. & Res. & Pred. & Res. & Pred. & Res. \\
\hline 1 & 1.342 & 1.192 & 0.15 & 1.148 & 0.194 & 1.125 & 0.217 & 1.225 & 0.117 \\
\hline 2 & 0.612 & 0.958 & -0.346 & 0.586 & 0.026 & 0.631 & -0.019 & 0.517 & 0.095 \\
\hline 3 & 0.38 & 0.418 & -0.038 & 0.341 & 0.039 & 0.313 & 0.067 & 0.354 & 0.026 \\
\hline 4 & -0.07 & -0.018 & -0.052 & -0.031 & -0.039 & -0.025 & -0.045 & -0.048 & -0.022 \\
\hline 5 & 0.819 & 0.851 & -0.032 & 0.795 & 0.024 & 0.834 & -0.015 & 0.763 & 0.056 \\
\hline 6 & 1.255 & 1.285 & -0.03 & 1.057 & 0.198 & 1.107 & 0.148 & 1.074 & 0.181 \\
\hline 7 & 2.079 & 2.176 & -0.097 & 1.928 & 0.151 & 1.862 & 0.217 & 1.897 & 0.182 \\
\hline 8 & 1.633 & 1.582 & 0.051 & 1.475 & 0.158 & 1.487 & 0.146 & 1.482 & 0.151 \\
\hline 9 & 1.176 & 1.245 & -0.069 & 1.057 & 0.119 & 1.182 & -0.006 & 1.116 & 0.06 \\
\hline 10 & 1.204 & 1.235 & -0.031 & 1.152 & 0.052 & 1.194 & 0.01 & 1.263 & -0.059 \\
\hline 11 & 1.838 & 1.907 & -0.069 & 1.789 & 0.049 & 1.812 & 0.026 & 1.749 & 0.089 \\
\hline 12 & 1.903 & 1.986 & -0.083 & 1.745 & 0.158 & 1.782 & 0.121 & 1.856 & 0.047 \\
\hline 13 & 0.698 & 0.729 & -0.031 & 0.595 & 0.103 & 0.682 & 0.016 & 0.707 & -0.009 \\
\hline 14 & 1.431 & 1.45 & -0.019 & 1.362 & 0.069 & 1.382 & 0.049 & 1.332 & 0.099 \\
\hline 15 & 1.322 & 1.371 & -0.049 & 1.263 & 0.059 & 1.206 & 0.116 & 1.194 & 0.128 \\
\hline 16 & 1.518 & 1.582 & -0.064 & 1.404 & 0.114 & 1.318 & 0.2 & 1.354 & 0.164 \\
\hline 17 & 0.146 & 0.174 & -0.028 & 0.119 & 0.027 & 0.034 & 0.112 & 0.163 & -0.017 \\
\hline 18 & 0.556 & 0.465 & 0.091 & 0.383 & 0.173 & 0.364 & 0.192 & 0.533 & 0.023 \\
\hline 19 & 1.041 & 0.987 & 0.054 & 0.964 & 0.077 & 1.026 & 0.015 & 1.132 & -0.091 \\
\hline 20 & 0.886 & 0.914 & -0.028 & 0.803 & 0.083 & 0.795 & 0.091 & 0.839 & 0.047 \\
\hline 21 & 0.301 & 0.288 & 0.013 & 0.219 & 0.082 & 0.291 & 0.01 & 0.232 & 0.069 \\
\hline 22 & 0.079 & 0.112 & -0.033 & 0.087 & -0.008 & 0.016 & 0.063 & 0.017 & 0.062 \\
\hline 23 & 0.748 & 0.719 & 0.029 & 0.663 & 0.085 & 0.618 & 0.13 & 0.706 & 0.042 \\
\hline 24 & 2.060 & 2.137 & -0.077 & 1.896 & 0.164 & 1.923 & 0.137 & 1.904 & 0.156 \\
\hline 25 & 2.000 & 1.899 & 0.101 & 1.938 & 0.062 & 2.043 & -0.043 & 1.813 & 0.187 \\
\hline 26 & 0.505 & 0.489 & 0.016 & 0.446 & 0.059 & 0.382 & 0.123 & 0.516 & -0.011 \\
\hline 27 & 0.763 & 0.786 & -0.023 & 0.722 & 0.041 & 0.615 & 0.148 & 0.679 & 0.084 \\
\hline 28 & -0.13 & -0.069 & -0.061 & -0.075 & -0.055 & -0.098 & -0.032 & -0.112 & -0.018 \\
\hline 29 & 1.23 & 1.336 & -0.106 & 1.29 & -0.06 & 1.295 & -0.065 & 1.166 & 0.064 \\
\hline 30 & 1.146 & 1.186 & -0.04 & 1.023 & 0.123 & 1.165 & -0.019 & 1.121 & 0.025 \\
\hline 31 & 0.755 & 0.693 & 0.062 & 0.614 & 0.141 & 0.579 & 0.176 & 0.726 & 0.029 \\
\hline 32 & 0.977 & 0.931 & 0.046 & 0.865 & 0.112 & 1.083 & -0.106 & 0.926 & 0.051 \\
\hline 33 & -0.031 & -0.016 & -0.015 & -0.059 & 0.028 & -0.021 & -0.01 & -0.071 & 0.04 \\
\hline 34 & 1.897 & 1.853 & 0.044 & 1.824 & 0.073 & 1.813 & 0.084 & 1.917 & -0.02 \\
\hline 35 & 1.778 & 1.793 & -0.015 & 1.681 & 0.097 & 1.691 & 0.087 & 1.644 & 0.134 \\
\hline 36 & 1.845 & 1.863 & -0.018 & 1.807 & 0.038 & 1.715 & 0.13 & 1.767 & 0.078 \\
\hline 37 & 1.000 & 0.944 & 0.056 & 1.042 & -0.042 & 0.959 & 0.041 & 0.911 & 0.089 \\
\hline 38 & 0.913 & 0.879 & 0.034 & 0.873 & 0.04 & 0.817 & 0.096 & 0.882 & 0.031 \\
\hline 39 & 0.462 & 0.387 & 0.075 & 0.398 & 0.064 & 0.487 & -0.025 & 0.408 & 0.054 \\
\hline 40 & 0.886 & 0.765 & 0.121 & 0.976 & -0.09 & 0.798 & 0.088 & 0.834 & 0.052 \\
\hline 41 & 0.505 & 0.486 & 0.019 & 0.349 & 0.156 & 0.518 & -0.013 & 0.471 & 0.034 \\
\hline 42 & 0.255 & 0.218 & 0.037 & 0.181 & 0.074 & 0.272 & -0.017 & 0.164 & 0.091 \\
\hline 43 & 1.23 & 1.183 & 0.047 & 1.072 & 0.158 & 1.363 & -0.133 & 1.15 & 0.08 \\
\hline 44 & 0.662 & 0.653 & 0.009 & 0.617 & 0.045 & 0.591 & 0.071 & 0.585 & 0.077 \\
\hline 45 & 0.462 & 0.421 & 0.041 & 0.269 & 0.193 & 0.327 & 0.135 & 0.363 & 0.099 \\
\hline 46 & 1.401 & 1.372 & 0.029 & 1.279 & 0.122 & 1.312 & 0.089 & 1.366 & 0.035 \\
\hline 47 & 1.079 & 1.024 & 0.055 & 0.947 & 0.132 & 0.994 & 0.085 & 0.961 & 0.118 \\
\hline 48 & 0.69 & 0.641 & 0.049 & 0.624 & 0.066 & 0.608 & 0.082 & 0.586 & 0.104 \\
\hline 49 & 1.041 & 1.169 & -0.128 & 0.931 & 0.11 & 0.949 & 0.092 & 0.879 & 0.162 \\
\hline 50 & 2.041 & 1.954 & 0.087 & 1.883 & 0.158 & 1.963 & 0.078 & 1.913 & 0.128 \\
\hline
\end{tabular}


TABLE 3: Continued.

\begin{tabular}{lccccccccc}
\hline \multirow{2}{*}{ Com } & \multirow{2}{*}{$\mathrm{pIC}_{50}$} & \multicolumn{2}{c}{ G-QSAR model 1 } & \multicolumn{2}{c}{ G-QSAR model 2 } & \multicolumn{2}{c}{ G-QSAR model 3 } & \multicolumn{2}{c}{ SA-kNN model 4 } \\
& & Pred. & Res. & Pred. & Res. & Pred. & Res. & Pred. & Res. \\
\hline 51 & 1.361 & 1.317 & 0.044 & 1.376 & -0.015 & 1.227 & 0.134 & 1.183 \\
52 & 1.079 & 1.134 & -0.055 & 1.121 & -0.042 & 0.964 & 0.115 & 0.868 & 0.178 \\
53 & 2.69 & 2.631 & 0.059 & 2.465 & 0.225 & 2.539 & 0.151 & 2.576 & 0.114 \\
54 & 1.832 & 1.798 & 0.034 & 1.763 & 0.069 & 1.782 & 0.05 & 1.693 \\
55 & 1.505 & 1.463 & 0.042 & 1.361 & 0.144 & 1.476 & 0.029 & 1.361 & 0.139 \\
\hline
\end{tabular}

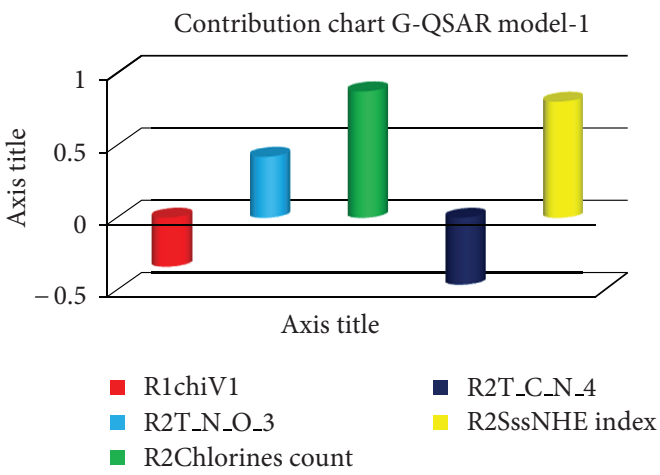

(a)

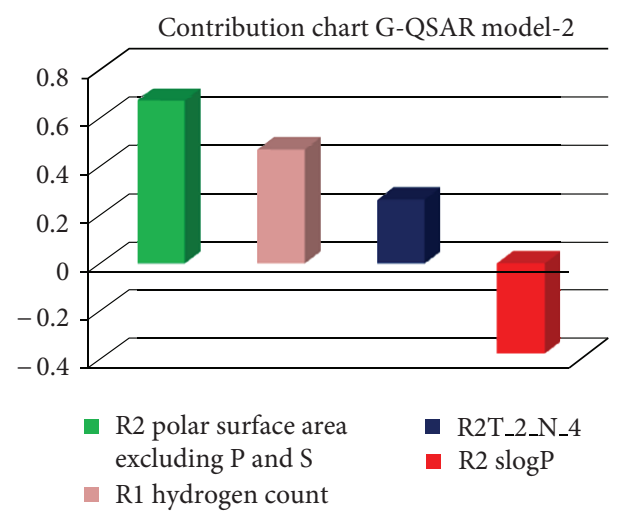

(c)

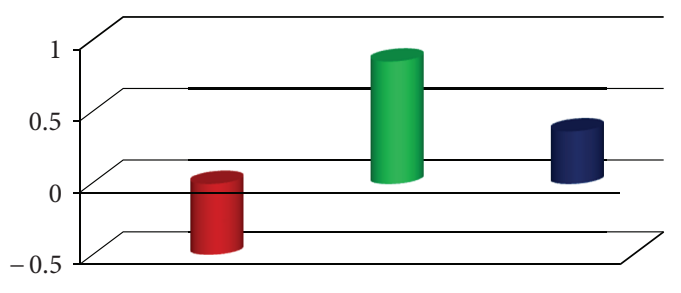

- R2SsSHcount

- R1SdSCHcount

- R2SddsN (nitro) count

(e)

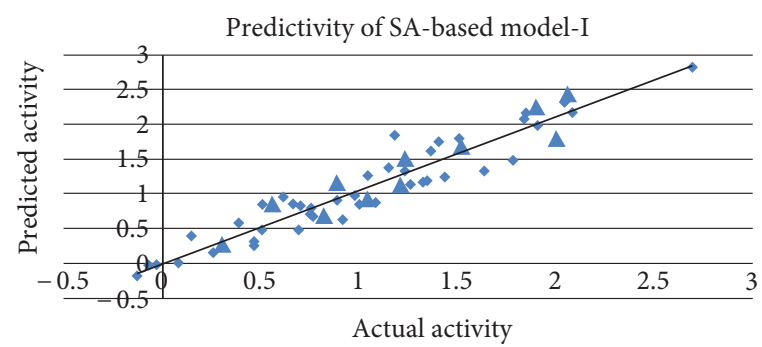

$\Delta$ Training set

- Test set

(b)

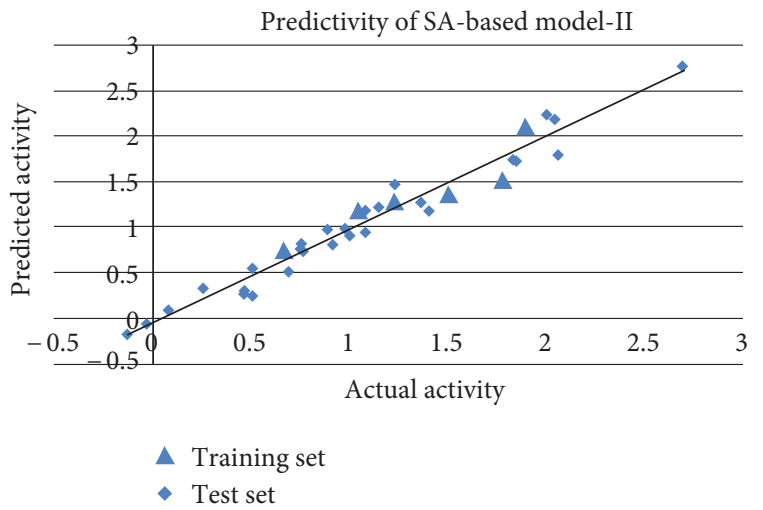

(d)

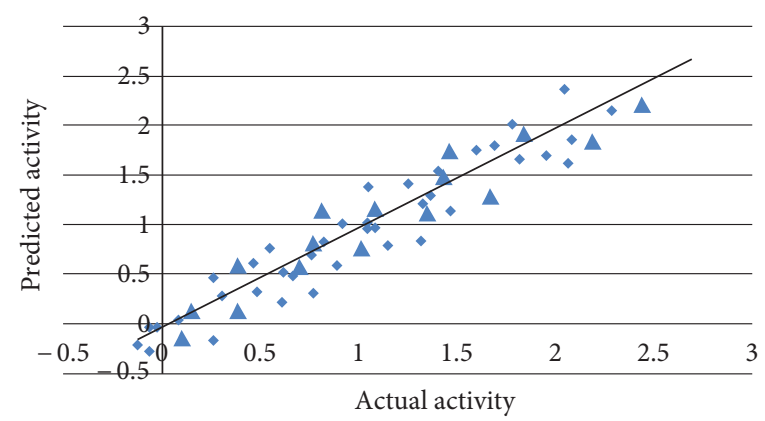

A Training set

- Test set

(f)

FIGURE 2: (a) Contribution charts of the descriptors for the model 1. (b) Graph of Actual and predicted activities of the training and test set GQSAR model 1 ( -Training set, $\Delta$-test set). (c) Contribution charts of the descriptors for the model 2. (d) Graph of Actual and predicted activities of the training and test set GQSAR model 2 ( $\bullet$-Training set, $\mathbf{\Delta}$-test set). (e) Contribution charts of the descriptors for the model 3. (f) Graph of Actual and predicted activities of the training and test set GQSAR model 3 ( $\bullet$-Training set, $\mathbf{\Delta}$-test set). 
the activity. The contributing descriptor in part- $\mathrm{R}_{2}$ is $\mathrm{R} 2$ polar surface area excluding $\mathrm{P}$ and $\mathrm{S}$ (i.e., phosphorus and sulfur) plays a most important role in determining activity in fragment R2. This descriptor signifies the total polar surface area excluding phosphorus and sulphur. This suggests that substituents such as $-\mathrm{OH}$ and $-\mathrm{COOH}$ would increase the antihypertensive activity. The descriptor R2SlogP in model represents to signify log of the octanol/water partition coefficient (including implicit hydrogen) which is a measure of the lipophilicity of the molecule. The descriptor is negatively correlated with biological activity in the QSAR models which suggests that decreasing the lipophilicity of the compounds will lead to increase in the antihypertensive activity. The activity contribution chart for GQSAR model is shown in Figure 2(c) and plots of observed versus predicted values of $\mathrm{pIC}_{50}$ are shown in Figure 2(d). The predicted (LOO) activities of the compounds by the above models is shown in Table 3.

$\mathrm{pIC}_{50}=-0.4874( \pm 0.0763)$ R2SsSHcount +0.8435 $( \pm 0.0543)$ R1SdsCHcount $+0.3623( \pm 0.2569)$ R2SddsN (nitro) count +0.0069 .

$N_{\text {traning }}=40, N_{\text {test }}=15, \mathrm{DF}=31, r^{2}=0.6472, q^{2}=0.5521$, $F$ test $=18.746, r^{2} \_$se $=0.3984, q^{2} \_$se $=0.2085$, pred $\_r^{2}=$ 0.6084 , pred_r $r^{2}$ se $=0.3781$.

Model 3 shows good squared correlation coefficient $\left(r^{2}\right)$ of 0.6472 explains $64 \%$ variance in biological activity. The low standard error of $r^{2} \_$se $=0.3984$ demonstrates accuracy of the model. This model also indicates statistical significance $>99.9 \%$ with $F$ values $F=18.746$. Cross validated squared correlation coefficient of this model was 0.5521 , which shows the good internal prediction power of this model. Another parameter for predictivity of test set compound is high pred_r $r^{2}=0.6084$ and low pred_r $r^{2}$ se $=0.3781$, which is showing good external predictive power of the model. The contributing descriptor in part- $\mathrm{R}_{1}$ is $\mathrm{R} 1 \mathrm{SdsCH}$ count indices for number of $-\mathrm{CH}$ group connected with one double and one single bond in a molecule. This is the positively contributing toward antihypertensive activity and it contributes approx $12 \%$. It suggests that increase in length of $-\mathrm{CH}$ atoms chain on that substitution site is favorable for the activity. Model 3 reveals that the contributing descriptor in part$\mathrm{R} 1$ is descriptor R2SsSHcount plays most important role with $(\sim 36 \%)$ the total number of -SH group connected with one single bond in determining antihypertensive activity. The next most important factor influencing activity variation is R2SddsN (nitro) count in the molecule and is inversely proportional to the activity and suggests the total number of nitro group connected with one single and two double bonds $\mathrm{R}^{2}$ position will lead to improved antihypertensive activity. The activity contribution chart for QSAR model is shown in Figure 2(e) and plots of observed versus predicted values of $\mathrm{pIC}_{50}$ are shown in Figure 2(f). The predicted (LOO) activities of the compounds by the above models are shown in Table 3.

For a better understanding of the QSAR models of such $1,-2,-4$ triazolinone compounds, an attempt to generate a 3D-QSAR models has also been made. The kNN MFA QSAR method explores formally the active analogue approach which implies that compounds display similar profiles of pharmacological activities. In this method the activity of each compound is predicted as average activity of $k$ most chemically similar compounds from that data set. The predictive ability of this simulated annealing $k$-nearest neighbour MFA model was evaluated by predicting the biological activities of the test set molecules.

$\mathrm{pIC}_{50}=-1.8421-\mathrm{S} \_305(-0.0182,-0.0112)+\mathrm{H} \_1193$ $(0.2863,0.3924)-$ S_381 $(-0.0640,-0.0344)-\bar{E} \_430$ $(-8.6560,-2.3329)$.

$k$-nearest neighbour $=4, N_{\text {traning }}=40, N_{\text {test }}=15, \mathrm{DF}=38$, $q^{2}=0.7461, F$ test $=69.631, q^{2} \_$se $=0.4863$, pred_ $r^{2}=0.7790$, pred_r $r^{2}$ se $=0.3047, Z$ Score $\mathrm{Q}^{2}=3.7446$.

The $k$-Nearest Neighbour-MFA QSAR method explores formally the active analogue approach which implies that compounds display similar profiles of pharmacological activities. In this method, the activity of each compound is predicted as average activity of $k$ most chemically similar compounds from the data set. The predictive ability of this simulated annealing $k$-nearest neighbour-MFA model was evaluated by predicting the biological activities of the test set molecules. For the series of $3 \mathrm{H}-1,-2,-4$ triazolinone aryl and nonaryl substituents, the template-based model yielded validation $\left(q^{2}\right)$ and cross validation (pred_ $\left.r^{2}\right)$ values of 0.7461 and 0.7790 , respectively. The LOO cross-validated analysis of the best model gave rise to a $q^{2}$ value of 0.7461 suggesting that the model is a useful tool for predicting anti-hypertensive activities. The correlation coefficient between the calculated and experimental activities amounts to a value of 0.8036 with an SE of 0.1895. The descriptors S_305, H_1193, S_381, and E_430 are the steric, electrostatic, and hydrophobic field energy of interactions between probe $\left(\mathrm{CH}_{3}\right)$ and compounds at their corresponding spatial grid points of 305, 1193, 381, and 430. These points suggested the significance and requirement of hydrophobic and steric properties as mentioned in the ranges in parenthesis for SAR and maximum biological activities of triazolinone analogues. The graphical representations and model summary of $k$-nearest neighbourMFA results for angiotensin II $\mathrm{AT}_{1}$ antagonists inhibitors are shown in Figure 3(a). The predicted activities of the compounds by the above model are shown in Table 3 . In 3D-QSAR studies, 3D data points generated triazolinone pharmacophore were used to optimize the electrostatic and steric requirements of the triazolinone nucleus for the antihypertensive activity. From 3D-QSAR model, it is observed that electrostatic descriptor like E_430 (-8.6560, - 2.3329) which indicates that substitution involving electron deficient group is preferred for substitution at R2 position, the chlorine and nitro substituted compound can show potent activity. The electrostatic interaction at lattice point E_430 is negatively contributing, which means substitution of electron withdrawing groups on the ring of triazolinone can increase the antihypertensive activity and hence less electronegative substituent group is preferred in that region. Other steric descriptors S_305 and S_381 with negative coefficients indicate the regions, where less bulky group substitutions are preferable for better antihypertensive activity. Positive values in field descriptor indicated the requirement of positive 


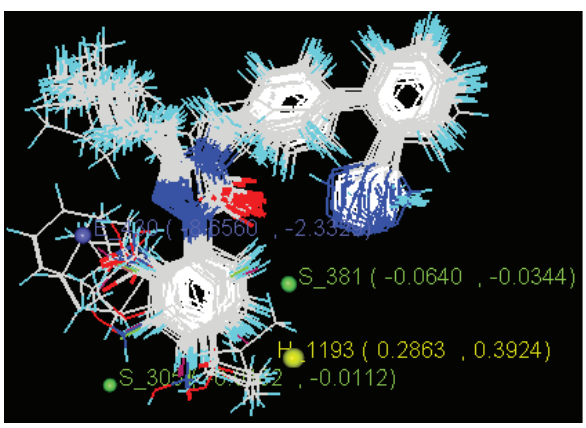

(a)

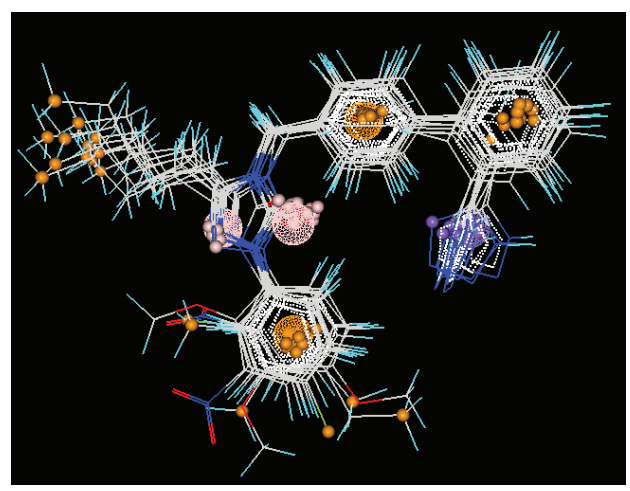

(c)

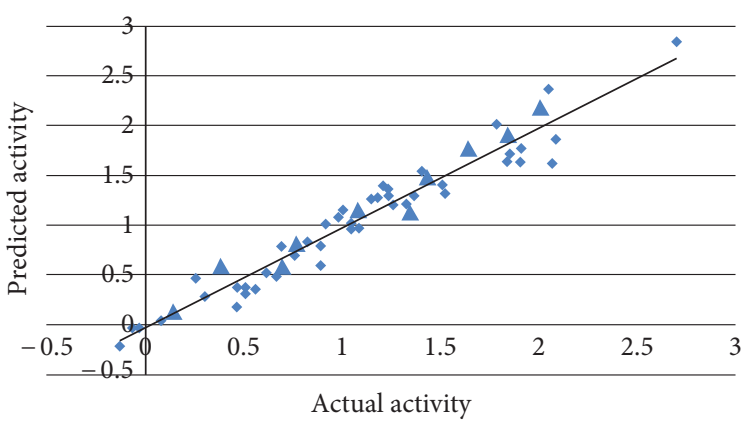

Training set

- Test set

(b)

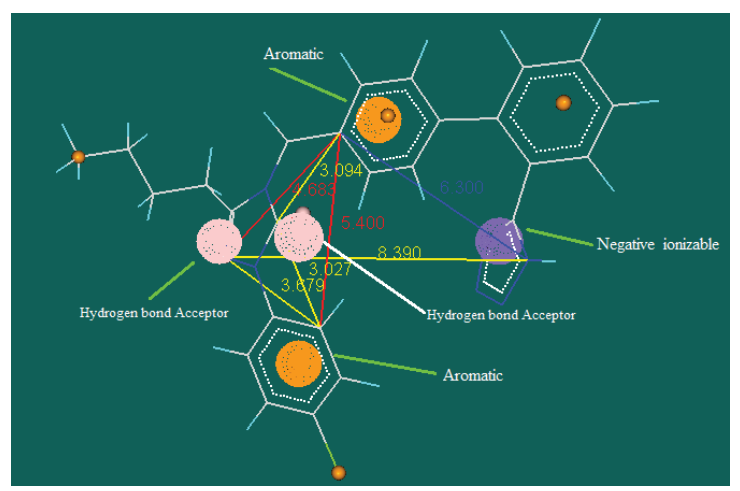

(d)

Figure 3: (a) Stereoview of the best SA-MLR corresponding to 3D model. (b) Graph of Actual and predicted activities of the training and test set 3D model ( $\bullet$-Training set, $\mathbf{\Delta - t e s t ~ s e t ) . ~ ( c ) ~ A l i g n e d ~ b i o p h a r m a c o p h o r e ~ m o d e l . ~ ( d ) ~ B e s t ~ d i s t a n c e ~ b i o p h a r m a c o p h o r e ~ m o d e l . ~}$

hydrophobic potential for enhancing the biological activity of triazolinone analogues. Therefore more hydrophobic substituents such as $-\mathrm{C}_{6} \mathrm{H}_{11},-\mathrm{C}_{6} \mathrm{H}_{5},-\mathrm{C}\left(\mathrm{CH}_{3}\right)_{3},-\mathrm{CF}_{3},-\mathrm{CH}_{3}$ [71] were preferred at the position of generated data point H_1193 around triazolinone moiety. The plot of actual versus predicted activity for the training and test sets of compounds the cases are represented in Figure 3(b). The plot of observed versus predicted activity provides an idea about how well the model was trained and how well it predicts the activity of the external test set.

The pharmacophore mapping was carried out to map the chemical features or functional groups present in the reported compounds and screened the new molecules from specs database. Selected pharmacophore shows five chemical features which were present in all aligned 55 molecules (Figure 3(c)). The information shows that the five features used were two HAc feature (hydrogen bond acceptor), two AroC feature (Aromatic), and one negative ionizable feature. The average RMSD of the pharmacophore alignment of each two molecules is $0.3174 \AA$. From distance geometry studies of the pharmacophore, it is clear that for optimum factor activity, the distance between the two hydrogen bond acceptor features should be about $4.683 \AA$ and $3.094 \AA$ while the distance between the aromatic feature in biphenyl moiety. Another distance between the hydrogen bond acceptor features and negative ionizable should be about $8.390 \AA$, while the distance between the hydrophobic and negative ionizable feature should be $6.300 \AA$ (Figure 3(d)).

\section{Conclusion}

Molecular modeling studies were performed to design new more potent compounds to inhibit $3 \mathrm{H}-1,-2,-4$ triazolinone aryl and nonaryl substituents for antihypertensive activity. In the present Group based QSAR studies investigation, all proposed QSAR models were statistically significant. However Group based QSAR model by coupled with simulated annealing with MLR a could be considered as best one in terms of excellent internal and external predictive abilities. $k$-nearest neighbour 3D QSAR was performed on the same series to correlate the effects of steric, electrostatic, and hydrophobic parameters with the activity using simulated annealing method. The findings of 3D QSAR studies provided the overall substitution pattern (electrostatic, steric, and hydrophobic fields) required around the $3 \mathrm{H}-1,-2,-4$ triazolinone aryl and nonaryl substituents. Furthermore, visualization of the 3DQSAR model in the context of the molecules under study provided details of the relationship between structure and activity and thus provides explicit indications for the design of better analogues. Pharmacophore mapping was also done on the conformers of the triazolinone compounds generated 
by the MolSign. Furthermore, we hope that the current study provides better insight into the designing of more potent angiotensin II $\mathrm{AT}_{1}$ antagonists as antihypertensive agent in the future before their synthesis.

\section{Acknowledgments}

The authors would like to thank journal referees for their valuable suggestions to improve the paper. They thank VLife Sciences Technologies Private Limited Pune, India for providing trial version of the software.

\section{References}

[1] I. H. Page and O. M. Helmer, "A crystalline pressor substance (angiotonin) resulting from the reaction between renin and renin activator," The Journal of Experimental Medicine, vol. 71, pp. 29-42, 1940.

[2] E. Braun-Menendez, E. Fasciolo, J. C. Leloir, and J. M. Munoz, "The substance causing renal hypertension," The Journal of Physiology, vol. 98, pp. 283-298, 1940.

[3] C. M. Ferrario, "The renin-angiotensin system: importance in physiology and pathology," Journal of Cardiovascular Pharmacology, vol. 15, supplement 3, no. 3, pp. S1-S5, 1990.

[4] M. B. Vallotton, "The renin-angiotensin system," Trends in Pharmacological Sciences, vol. 8, no. 2, pp. 69-74, 1987.

[5] M. De Gasparo, K. J. Catt, T. Inagami, J. W. Wright, and T. Unger, "International union of pharmacology. XXIII. The angiotensin II receptors," Pharmacological Reviews, vol. 52, no. 3, pp. 415-472, 2000.

[6] M. De Gasparo, "AT 1 and $\mathrm{AT}_{2}$ angiotensin II receptors: key features," Drugs, vol. 62, no. 1, pp. 1-10, 2002 (French).

[7] D. F. Guo, Y. L. Sun, P. Hamet, and T. Inagami, "The angiotensin II type 1 receptor and receptor-associated proteins," Cell Research, vol. 11, no. 3, pp. 165-180, 2001.

[8] M. M. Ibrahim, "RAS inhibition in hypertension," Journal of Human Hypertension, vol. 20, no. 2, pp. 101-108, 2006.

[9] U. Gether, "Uncovering molecular mechanisms involved in activation of G protein-coupled receptors," Endocrine Reviews, vol. 21, no. 1, pp. 90-113, 2000.

[10] D. T. Dinh, A. G. Frauman, C. I. Johnston, and M. E. Fabiani, "Angiotensin receptors: distribution, signalling and function," Clinical Science, vol. 100, no. 5, pp. 481-492, 2001.

[11] A. Parate and S. C. Chaturvedi, "Predicting 3H-1,2,4triazolinones as angiotensin II receptor antagonists: 2D and 3D QSAR by kNN-molecular field analysis approach," Medicinal Chemistry Research, vol. 21, no. 7, pp. 1166-1178, 2011.

[12] C. Hansch, "A quantitative approach to biochemical structureactivity relationships," Accounts of Chemical Research, vol. 2, no. 8, pp. 232-239, 1969.

[13] A. M. Doweyko, “QSAR: dead or alive?” Journal of ComputerAided Molecular Design, vol. 22, no. 2, pp. 81-89, 2008.

[14] M. C. Sharma, D. V. Kohli, S. C. Chaturvedi, and S. Sharma, "Molecular modelling studies of some substitued 2butylbenzimidazoles angiotensin II receptor antagonists as antihypertensive agents," Digest Journal of Nanomaterials and Biostructures, vol. 4, no. 4, pp. 843-856, 2009.

[15] M. C. Sharma and D. V. Kohli, "3D QSAR studies on series of 2, 3-dihydro-4(1H)-quinazolinone derivatives angiotensin II receptor antagonists: kNNMFA approach," American-Eurasian
Journal of Agricultural \& Environmental Sciences, vol. 6, no. 2, pp. 85-94, 2011.

[16] M. C. Sharma and D. V. Kohli, "3D QSAR kNNMFA approach studies on series of substituted piperidin-2-one biphenyl tetrazoles as angiotensin II receptor antagonists," AmericanEurasian Journal of Toxicological Sciences, vol. 3, no. 2, pp. 75-84, 2011.

[17] M. C. Sharma and D. V. Kohli, "QSAR study on sulfonylcarbamate derivatives: an insight into the structural requirement for the angiotensin II receptor antagonist," European Journal of Applied Sciences, vol. 3, no. 1, pp. 9-14, 2011.

[18] M. C. Sharma and D. V. Kohli, "3D-QSAR studies of some substituted imidazolinones angiotensin II receptor antagonists," World Applied Sciences Journal, vol. 12, no. 11, pp. 2129-2134, 2011.

[19] M. C. Sharma and D. V. Kohli, "3D QSAR studies on a series of-[(1- Benzyl-1H-Imidazol-5-yl)-alkyl]-amino derivatives as angiotensin II AT1 antagonists," American-Eurasian Journal of Agricultural \& Environmental Sciences, vol. 6, no. 2, pp. 79-84, 2011.

[20] M. C. Sharma and D. V. Kohli, "3D QSAR approach on substituted isoxazolidines derivatives as angiotensin II receptor antagonist," American-Eurasian Journal of Toxicological Sciences, vol. 3, no. 2, pp. 85-91, 2011.

[21] M. C. Sharma and D. V. Kohli, " 3 D QSAR studies of substituted- $4(3 \mathrm{H})$ quinazolinones derivatives as angiotensin II receptor antagonists," European Journal of Applied Sciences, vol. 3, no. 1, pp. 15-23, 2011.

[22] M. C. Sharma and D. V. Kohli, "Predicting alkylbenzimidazole derivatives as angiotensin II receptor antagonists: 3D QSAR by kNN-MFA approach," Advanced Biology Research, vol. 5, no. 3, pp. 161-169, 2011.

[23] M. C. Sharma and D. V. Kohli, “3D QSAR studies on a series of sulfonylcarbamate isostere derivatives as nonpeptide angiotensin II receptor antagonists: kNNMFA method," American-Eurasian Journal of Agricultural \& Environmental Sciences, vol. 6, no. 2, pp. 64-70, 2011.

[24] M. C. Sharma and D. V. Kohli, "Exploration of quantitative structure activity relationship studies on a series of substituted quinazolinones as angiotensin II AT1 receptor antagonists," World Applied Sciences Journal, vol. 12, no. 11, pp. 2111-2119, 2011.

[25] M. C. Sharma and D. V. Kohli, "2D- and 3D- QSAR studies of substituted $4 \mathrm{H}$-pyrido [1,2-a] pyrimidin-4-ones angiotensin II receptor antagonists," American-Eurasian Journal of Agricultural \& Environmental Sciences, vol. 3, no. 2, pp. 92-100, 2011.

[26] M. C. Sharma and D. V. Kohli, "An insight into the structural requirement QSAR approach on substituted isoxazolidines derivatives as angiotensin II receptor antagonist," AmericanEurasian Journal of Agricultural \& Environmental Sciences, vol. 6, no. 2, pp. 71-78, 2011.

[27] M. C. Sharma and D. V. Kohli, "QSAR studies on substituted benzimidazoles as angiotensin II receptor antagonists: $\mathrm{kNN}$ MFA approach," Arabian Journal of Chemistry. In press.

[28] M. C. Sharma and D. V. Kohli, "An approach to design antihypertensive agents by $2 \mathrm{D}$ QSAR studies on series of substituted benzimidazoles derivatives as angiotensin II receptor antagonists," Arabian Journal of Chemistry. In press.

[29] M. C. Sharma and and D. V. Kohli, "QSAR analysis and 3D QSAR kNN-MFA approach on a series of substituted quinolines derivatives as angiotensin II receptor antagonists," Arabian Journal of Chemistry. In press. 
[30] M. C. Sharma and D. V. Kohli, "Quantitative structure-activity analysis studies on triazolinone aryl and nonaryl substituents as angiotensin II receptor antagonists," Journal of Saudi Chemical Society. In press.

[31] M. C. Sharma and D. V. Kohli, “Two dimensional and k-Nearest neighbor molecular field analysis approach on substituted triazolone derivatives: an insight into the structural requirement for the angiotensin II receptor antagonist," Journal of Saudi Chemical Society. In press.

[32] M. C. Sharma and D. V. Kohli, "Insight into the structural requirement of substituted quinazolinone biphenyl acylsulfonamides derivatives as angiotensin II receptor antagonists: 2D and 3D QSAR approach," Journal of Saudi Chemical Society. In press.

[33] M. C. Sharma and D. V. Kohli, "QSAR analysis of imidazo[4, 5-b] pyridine substituted á-phenoxyphenylacetic acids as angiotensin II AT1 receptor antagonists," Journal of Saudi Chemical Society. In press.

[34] M. C. Sharma and D. V. Kohli, "Predicting substituted 2butylbenzimidazoles derivatives as angiotensin II receptor antagonists: three-dimensional QSAR and pharmacophore mapping," Journal of Saudi Chemical Society. In press.

[35] M. C. Sharma and D. V. Kohli, "QSAR studies of a series of angiotensin II receptor substituted benzimidazole bearing acidic heterocycles derivatives," Journal of Saudi Chemical Society. In press.

[36] M. C. Sharma, S. Sharma, N. K. Sahu, and D. V. Kohli, "3D QSAR kNNMFA studies on 6-substituted benzimidazoles derivatives as nonpeptide angiotensin II receptor antagonists: a rational approach to antihypertensive agents," Journal of Saudi Chemical Society. In press.

[37] M. C. Sharma, S. Sharma, N. K. Sahu, and D. V. Kohli, "QSAR Studies of some substituted imidazolinones angiotensin II receptor antagonist using partial least squares regression (PLSR) based feature selection," Journal of Saudi Chemical Society. In press.

[38] M. C. Sharma and D. V. Kohli, "Insight into the structural requirement of aryltriazolinone derivatives as angiotensin II AT1 receptor: 2D and 3D-QSAR k-nearest neighbor molecular field analysis approach," Medicinal Chemistry Research, vol. 21, pp. 2837-2853, 2012.

[39] M. C. Sharma and D. V. Kohli, "A comprehensive structureactivity analysis 2, 3,5-trisubstituted 4,5-dihydro-4-oxo-3Himidazo [4,5-c] pyridine derivatives as angiotensin II receptor antagonists: using 2D- and 3D-QSAR approach," Medicinal Chemistry Research. In press.

[40] M. C. Sharma and D. V. Kohli, "Comprehensive two and three-dimensional QSAR studies of 3-substituted 6-butyl-1, 2dihydropyridin-2-ones derivatives as angiotensin II receptor antagonists," Medicinal Chemistry Research. In press.

[41] M. C. Sharma and D. V. Kohli, "Comprehensive structureactivity relationship analysis of isoxazolinyl and isoxazolidinyl substituted quinazolinone derivatives as angiotensin II receptor antagonists," Journal of Saudi Chemical Society. In press.

[42] M. C. Sharma, S. Sharma, and D. V. Kohli, "Structural insights for 5-â ketosulfoxide imidazolyl biphenyl sulfonylureas derivatives as angiotensin II AT1 receptor antagonists using kNNMFA with genetic algorithm," Journal of Saudi Chemical Society. In press.

[43] M. C. Sharma and D. V. Kohli, "A comprehensive structureactivity analysis of 5-carboxyl imidazolyl biphenyl sulfonylureas derivatives angiotensin AT1 receptor antagonists: 2D- and 3DQSAR approach," Arabian Journal of Chemistry. In press.

[44] M. C. Sharma, "Structural insight for (6-oxo-3-pyridazinyl)benzimidazoles derivatives as angiotensin II receptor antagonists: QSAR, pharmacophore identification and kNNMFA approach," Journal of Saudi Chemical Society. In press.

[45] M. C. Sharma and D. V. Kohli, "Comprehensive structureactivity relationship analysis of substituted 5-(biphenyl-4ylmethyl) pyrazoles derivatives as AT1 selective angiotensin II receptor antagonists: 2D and kNNMFA QSAR approach," Medicinal Chemistry Research. In press.

[46] M. C. Sharma, S. Sharma, and D. V. Kohli, "QSAR approach insight the structural requirement of substituted quinazolinones derivatives as angiotensin II receptor antagonists," Oxidation Communication, vol. 3, pp. 694-707, 2012.

[47] M. C. Sharma, S. Sharma, and D. V. Kohli, "QSAR studies of 2-alkylbenzimidazole derivatives as angiotensin II receptor antagonists," Oxidation Communication, vol. 3, pp. 708-721, 2012.

[48] M. C. Sharma and D. V. Kohli, "Predicting 2, 3-dihydroquinazolinones derivatives as angiotensin II receptor antagonists: 2D QSAR approach," Oxidation Communication, vol. 3, pp. 721-734, 2012.

[49] P. A. Datar, P. V. Desai, and E. C. Coutinho, "A 3D-QSAR of angiotensin II (AT1) receptor antagonists based on receptor surface analysis," Journal of Chemical Information and Computer Sciences, vol. 44, no. 1, pp. 210-220, 2004.

[50] T. Tuccinardi, V. Calderone, S. Rapposelli, and A. Martinelli, "Proposal of a new binding orientation for nonpeptide AT1 antagonists: homology modeling, docking and three-dimensional quantitative structure-activity relationship analysis," Journal of Medicinal Chemistry, vol. 49, no. 14, pp. 4305-4316, 2006.

[51] L. L. Chang, W. T. Ashton, K. L. Flanagan et al., "Triazolinones as nonpeptide angiotensin II antagonists. 1. Synthesis and evaluation of potent 2,4,5-trisubstituted triazolinones," Journal of Medicinal Chemistry, vol. 36, no. 17, pp. 2558-2568, 1993.

[52] VLife MDS 3.5, "Molecular design suite. Vlife Sciences Technologies Pvt. Ltd.”, Pune, India, 2008, http://www.vlifesciences.com/.

[53] T. A. Halgren, "Merck molecular force field. III. Molecular geometries and vibrational frequencies for MMFF94," Journal of Computational Chemistry, vol. 17, no. 5-6, pp. 553-586, 1996.

[54] T. Scior, J. L. Medina-Franco, Q. T. Do, K. Martínez-Mayorga, J. A. Y. Rojas, and P. Bernard, "How to recognize and workaround pitfalls in QSAR studies: a critical review," Current Medicinal Chemistry, vol. 16, no. 32, pp. 4297-4313, 2009.

[55] A. Golbraikh and A. Tropsha, "Predictive QSAR modeling based on diversity sampling of experimental datasets for the training and test set selection," Journal of Computer-Aided Molecular Design, vol. 16, no. 5-6, pp. 357-369, 2002.

[56] S. Ajmani, K. Jadhav, and S. A. Kulkarni, "Group-based QSAR (G-QSAR): mitigating interpretation challenges in QSAR," QSAR and Combinatorial Science, vol. 28, no. 1, pp. 36-51, 2009.

[57] S. Ajmani, A. Agrawal, and S. A. Kulkarni, "A comprehensive structure-activity analysis of protein kinase B-alpha (Akt1) inhibitors," Journal of Molecular Graphics and Modelling, vol. 28, no. 7, pp. 683-694, 2010. 
[58] S. Ajmani, K. Jadhav, and S. A. Kulkarni, "Three-dimensional QSAR using the k-nearest neighbor method and its interpretation," Journal of Chemical Information and Modeling, vol. 46, no. 1, pp. 24-31, 2006.

[59] P. Ghosh and M. C. Bagchi, "QSAR modeling for quinoxaline derivatives using genetic algorithm and simulated annealing based feature selection," Current Medicinal Chemistry, vol. 16, no. 30, pp. 4032-4048, 2009.

[60] M. Clark, R. D. Cramer III, and O. N. Van, "Validation of the general purpose tripos 5.2 force field," Journal of Computational Chemistry, vol. 10, pp. 982-1012, 1989.

[61] J. Gasteiger and M. Marsili, "Iterative partial equalization of orbital electronegativity-a rapid access to atomic charges," Tetrahedron, vol. 36, no. 22, pp. 3219-3228, 1980.

[62] M. A. Sharaf, D. L. Illman, and B. R. Kowalski, Chemometrics, Chemical Analysis Series, Wiley, New York, NY, USA, 1986.

[63] K. S. Bhadoriya, M. C. Sharma, S. V. Jain, G. S. Raut, and J. R. Rananaware, "Three-dimensional quantitative structureactivity relationship (3D-QSAR) analysis and molecular docking-based combined in silico rational approach to design potent and novel TRPV1 antagonists," Medicinal Chemistry Research. In press.

[64] W. Zheng and A. Tropsha, "Novel variable selection quantitative structure-property relationship approach based on the knearest neighbor principle," Journal of Chemical Information and Computer Sciences, vol. 40, no. 1, pp. 185-194, 2000.

[65] S. Kirkpatrick, C. D. Gelatt, and M. P. Vecchi, "Optimization by simulated annealing," Science, vol. 220, no. 4598, pp. 671-680, 1983.

[66] N. Metropolis, A. W. Rosenbluth, M. N. Rosenbluth, A. H. Teller, and E. Teller, "Equation of state calculations by fast computing machines," The Journal of Chemical Physics, vol. 21, no. 6, pp. 1087-1092, 1953.

[67] L. X. Sun, Y. L. Xie, X. H. Song, J. H. Wang, and R. Q. Yu, “Cluster analysis by simulated annealing," Computers and Chemistry, vol. 18, no. 2, pp. 103-108, 1994.

[68] M. C. Sharma, S. Sharma, and K. S. Bhadoriya, "QSAR analyses and pharmacophore studies of tetrazole and sulfonamide analogs of imidazo[4, 5-b]pyridine using simulated annealing based feature selection," Journal of Saudi Chemical Society. In press.

[69] R. D. Cramer, D. E. Patterson, and J. D. Bunce, "Comparative molecular field analysis (CoMFA). 1. Effect of shape on binding of steroids to carrier proteins," Journal of the American Chemical Society, vol. 110, no. 18, pp. 5959-5967, 1988.

[70] M. N. Noolvi and H. M. Patel, "A comparative QSAR analysis and molecular docking studies of quinazoline derivatives as tyrosine kinase (EGFR) inhibitors: a rational approach to anticancer drug design," Journal of Saudi Chemical Society. In press.

[71] K. S. Bhadoriya, S. V. Jain, S. B. Bari, M. L. Chavhan, and K. R. Vispute, "3D-QSAR study of indol-2-yl ethanones derivatives as novel indoleamine 2, 3-dioxygenase (IDO) inhibitors," $E$ Journal of Chemistry, vol. 9, pp. 1753-1759, 2012. 

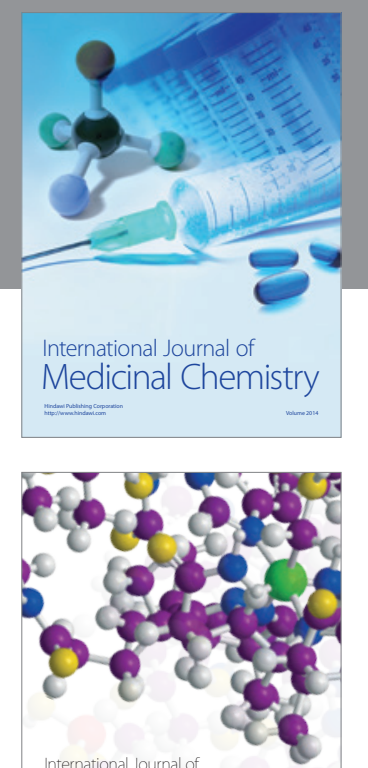

\section{Carbohydrate} Chemistry

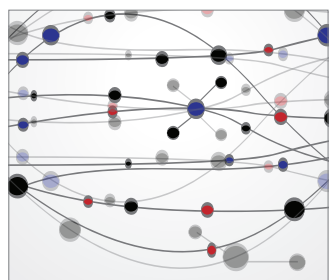

The Scientific World Journal
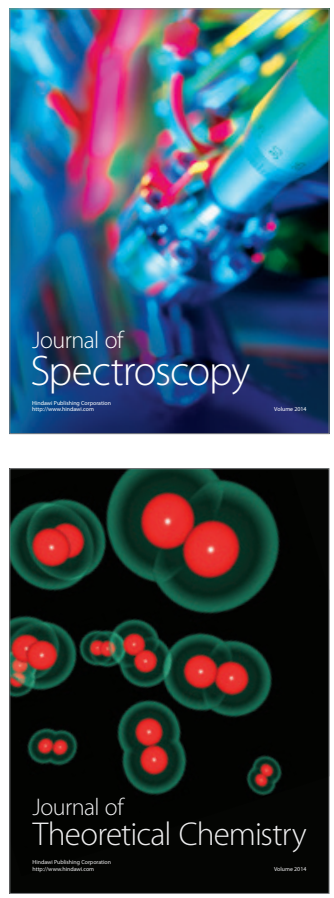
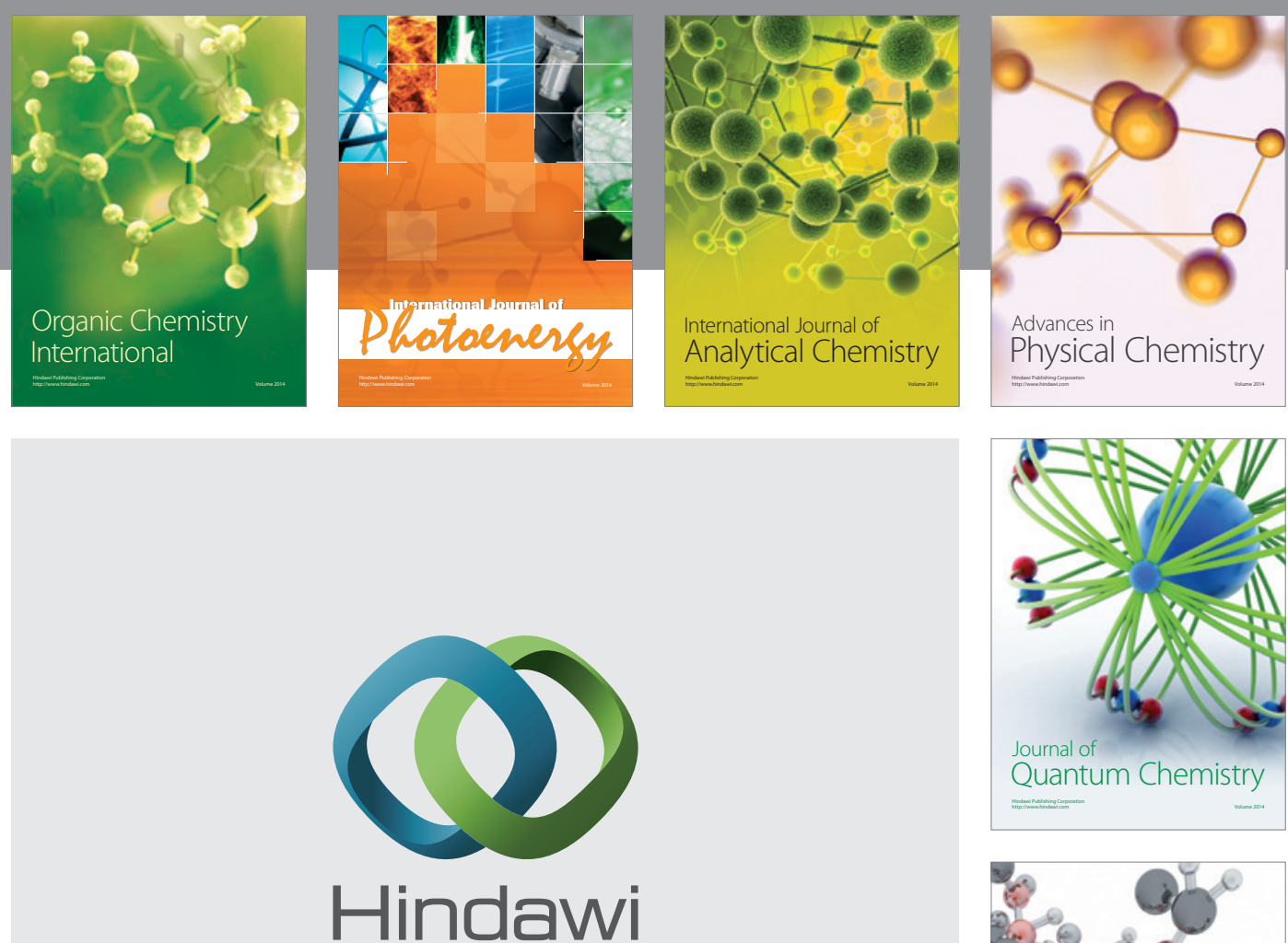

Submit your manuscripts at

http://www.hindawi.com

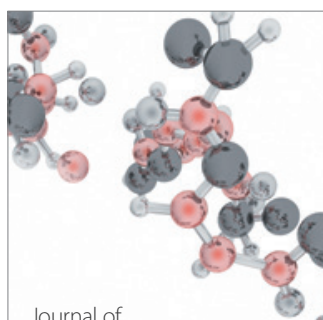

Analytical Methods

in Chemistry

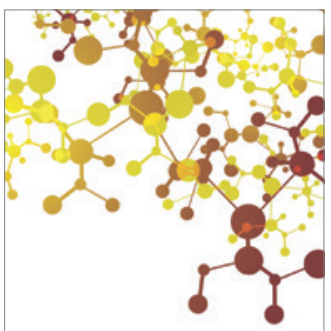

Journal of

Applied Chemistry

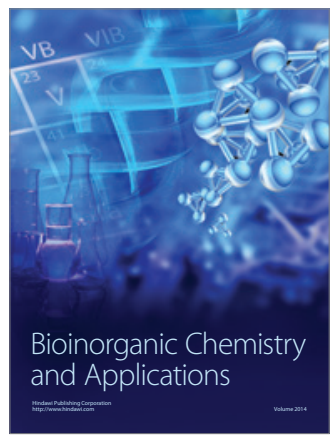

Inorganic Chemistry
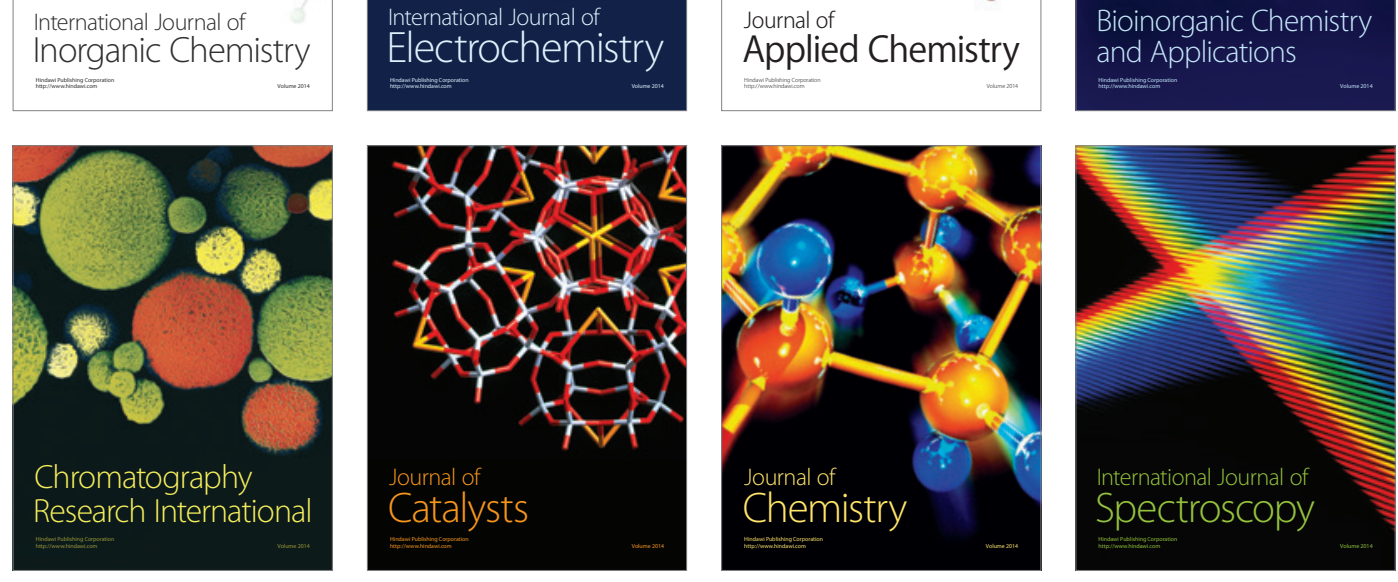